\title{
Factores para la implementación de cloud computing en empresas nuevas Caso Bogotá
}

\author{
CARLOS ANDRÉS OSORIO \\ Colegio de Estudios Superiores en Administración, CESA. \\ carloso@cesa.edu.co
}

Ingeniero Industrial MSc. Docente Investigador

\begin{abstract}
Resumen
En este documento se plantea el uso de cloud computing como un modelo para el manejo de los sistemas de información en empresas nacientes y los factores que más influyen en el momento de tomar la decisión de implementar su plataforma tecnológica sobre este modelo de acuerdo con estudios como los de Gartner. Se concentra en las empresas nuevas pues sus operaciones están por iniciar y los procesos de implementación de cloud computing son más sencillos al no tener que migrar la plataforma y los datos de sistemas legados a la "nube", lo que sería un proceso diferente y más complejo para las empresas que ya están funcionando. Para validar este planteamiento en el marco teórico, se presentan los resultados del estudio realizado a emprendedores de la incubadora de empresas de base tecnológica del SENA en Bogotá sobre su conocimiento del cloud computing y los factores a tener en cuenta para su implementación.
\end{abstract}

\section{Palabras Claves}

Cloud computing, emprendimiento, TIC, start up.

\section{Abstract}

This paper presents the use of cloud computing as a new model for managing information systems in start up projects and the most influential factors at the decision time to implement its platform technology on this model according to studies such as those of Gartner. It focuses on new businesses cause their operations are to begin and the process of implementation of cloud computing are easier to not have to migrate the platform and data from legacy systems to the "cloud", which would be a different process and more complex for companies that are already running. To validate the points made in the theoretical framework presents the results of the study of entrepreneurs in the incubator of technology companies SENA Bogotá about their knowledge of cloud computing and the factors to be considered for implementation.

\section{Key words}

Cloud computing, venturing, TIC, start up.
Fecha de recepción: 22 de abril de 2010

Fecha de aprobación: 14 de octubre de 2010

\section{Introducción}

La tecnología está evolucionando a gran velocidad haciendo que los equipos y los programas que posee una empresa queden obsoletos rápidamente, sin alcanzar a recuperar los costos de inversión. Un ejemplo de esto es la ley de Moore (Intel Corp.), la cual dice que la cantidad de circuitos en un transistor se duplica cada dos años; si miramos esto en las empresas que están iniciando, la inversión en tecnología es una de las primeras que se debe hacer y en muchas ocasiones una de las que más parte del presupuesto toma.

Como una nueva alternativa para esta situación aparece el cloud computing, que de acuerdo con Juan Manuel Rebés (2008) puede definirse como un

modelo inspirado en la idea de disponer de infraestructuras tecnológicas de modo que los recursos informáticos sean compartidos dinámicamente, se encuentren virtualizados y resulten accesibles como un servicio. Aúna de esta manera gran parte de las nuevas tendencias de software como servicio, virtualización de recursos, redes, grids e informática bajo demandla. En el modelo cloud computing, los grandes clusters de sistemas se enlazan entre sí para proporcionar servicios tecnológicos como si se tratase de un único superordenador global (Network World, 2008).

Esto abre una nueva alternativa a la forma de funcionamiento de las empresas, que al no depender de sistemas operativos específicos, amplía las opciones en el momento de tomar decisiones relacionadas con tecnologías de información (TI). Permite también dar movilidad a los empleados al tener acceso en 
cualquier sitio con conexión a internet y concentrar los recursos de la organización en sus factores críticos de éxito, evitando preocupaciones por el mantenimiento de sus sistemas de información (tanto hardware como software), al trasladar esta responsabilidad a proveedores externos de estas soluciones informáticas.

Por esto se propone la utilización de cloud computing como un modelo de negocio en el que al pagar por uso, se racionaliza el gasto en TIC permitiendo concentrar los recursos financieros y de personal en el core del negocio, convirtiéndose en un factor que apoya la creación y sostenimiento de las empresas, al disminuir la cantidad de dinero necesaria para el montaje de la empresa y con la promesa de una infraestructura robusta y confiable para la gestión de la información respecto a las necesidades básicas de manejo de datos de la empresa, pues solo se necesitan computadores de características normales y conexión a internet para el uso de los programas que necesiten.

De acuerdo con Dignan (2008), para 2012, el 80\% de las mil empresas Fortune pagarán por algún servicio de cloud computing y un 30\% de ellas pagará por infraestructura cloud computing; y para 2010, más del 80\% de las empresas usuarias de cloud computing, se dedicará a las consultas de datos muy grandes, a trabajos paralelos masivos de corta duración, o el uso de las tecnologías de la creación de una empresa con poca o ninguna infraestructura de TI. Lo que muestra el impulso de este modelo y su aplicación en el mundo empresarial en compañías de gran tamaño y alto reconocimiento, que al dar ellos la aprobación de esta tecnología por medio de su utilización validan la utilidad de este modelo para grandes empresas y por analogía a pequeñas empresas.

A continuación se presentan algunos conceptos para que al final del artículo las empresas puedan tomar la decisión sobre si cloud computing es una alternativa viable para su idea de negocio o no.

\section{Cloud computing}

De acuerdo con Miller (2009) con el cloud computing

en vez de correr programas y datos en computadores de escritorio tradícionales, todo estará almacenado en la "nube", entendida esta nube como un ensamble de computadores y servidores a los cualles se tiene acceso vía internet. Ell cloud computing le permite acceder a su información desde cualquier parte en el mundo, litberándolo de las fronteras de su computador de escritorio y facilitando la collaboración entre miembros del equipo en diferentes sitios.

Esto muestra una definición alternativa, que por medio de la aplicación permite formar una idea más clara de qué es cloud computing.

\section{Propiedades del cloud computing}

De acuerdo con Google (referenciado por Miller, 2009), el cloud computing tiene seis propiedades:

- Centrado en el usuario. Es decir una vez el usuario se conecta a la nube, todo lo que se almacene en ella se convierte en propio, no solo el documento o el archivo, sino el derecho a compartirlo con otros.

- Centrado en las tareas. En vez de concentrarse en la aplicación y que puede hacer ella, se concentra en que necesita tener listo el usuario y como la aplicación lo puede hacer por él. Las aplicaciones tradicionales están teniendo menos importancia que los documentos que ellas crean.

- Es poderosa. Pues conecta cientos de miles de computadores juntos en una nube que genera un poder computacional poco posible con un solo computador o supercomputador

- Es accesible. Pues los datos se almacenan en la nube y los usuarios pueden traer esta información e información adicional de otros repositorios. El usuario no está limitado a una sola fuente de datos como en los computadores de escritorio.

- Es inteligente. Con la variedad de datos almacenados en la nube, el data minning y el análisis son necesarios para acceder a la información de forma inteligente.

- Es programable. Varias de las tareas necesarias para el cloud computing son automatizadas. Por ejemplo, la protección de la integridad de los datos, replicación de los datos en otros computadores en el mundo, entre otros. 


\section{Estructura de funcionamiento}

El cloud computing se basa en la infraestructura existente de telecomunicaciones y en los protocolos web como se observa en el gráfico 1, en el que se trabaja un internet optimizado para hospedar las aplicaciones y el acceso a los servidores.

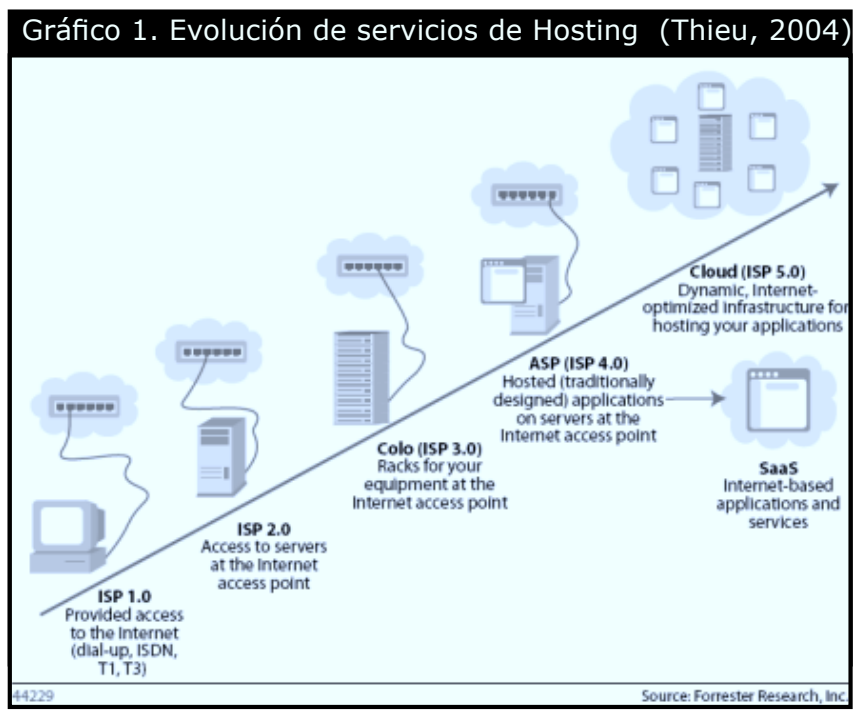

De manera más específica el modelo de cloud computing está centrado en la red que se representa como la nube (GRÁFICO 2). La ventaja de esta característica es que aprovecha la infraestructura instalada para la de distribución de internet a los usuarios.

Por medio de la red se acceden a servicios de almacenamiento y procesamiento de datos ubicados en diferentes partes del mundo, proceso que debe ser transparente para el usuario final y que de acuerdo con Perry (2008), tampoco debe ser cuestión de interés para este el interés es poder acceder desde cualquier dispositivo con conexión a internet (inclusive desde un Ipod con la aplicación Mobile me).

\section{Pros y contras}

Por la novedad que presenta el gráfico 2 es necesario presentar los pros y los contras de esta tecnología, para que los usuarios interesados puedan tener el panorama completo, y contar con más elementos para decidir si se ajusta a las necesidades de su negocio.
Gráfico 2. Componentes de cloud computing (J., 2009)

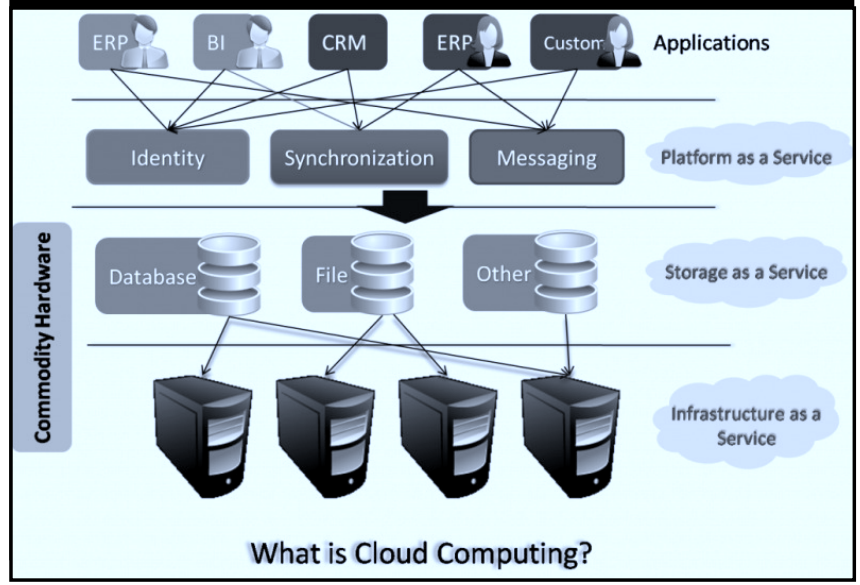

Como es normal con toda tecnología nueva se presentan muchos casos de éxito, en los que por lo general se exponen las ventajas de implementar soluciones cloud. Se recomienda visitar las páginas de amazon web services (Amazon) y de cloudTP (cloud $\mathrm{TP}$ ), en las que se recogen varios casos de empresas que han implementado cloud computing. CloudTP tiene una base más diversa de casos de estudio y casos de éxito. También se recomienda visitar (+ Publicidad \& marketing, 2010) para ver casos de éxito en Colombia.

Se hace la aclaración que los casos presentados en estas páginas tienden a brindar una versión optimista de las soluciones ofrecidas por estas empresas, por lo que parte de esa información debe tomarse con beneficio de inventario. En la búsqueda de artículos en contra se pueden encontrar artículos de (Lohr, 2001), columnista del New York Times y el de Richard Stallman (Johnson, 2008) publicado en el periódico Guardian de Inglaterra.

Se presenta primero un listado paralelo de pros y contras, para que se puedan comparar con facilidad y luego se amplía la información de estos (Tabla 1). 
Tabla 1. Pros y contras del cloud computing

\begin{tabular}{|c|c|}
\hline Pros & Contras \\
\hline 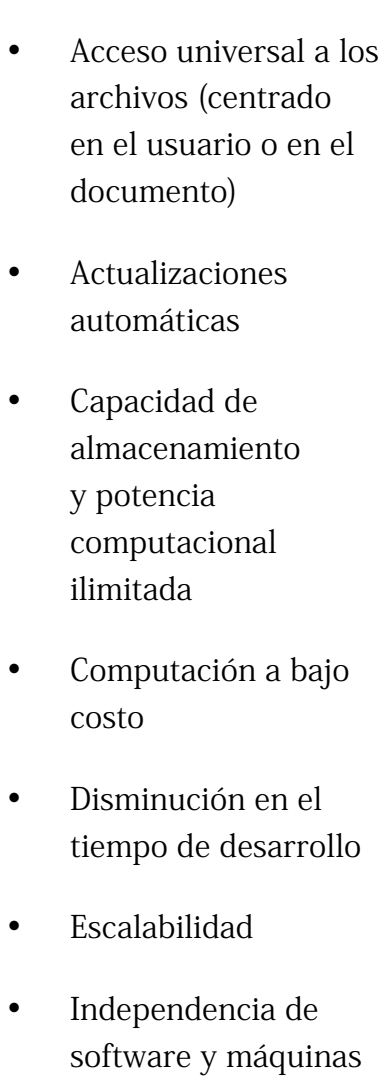 & 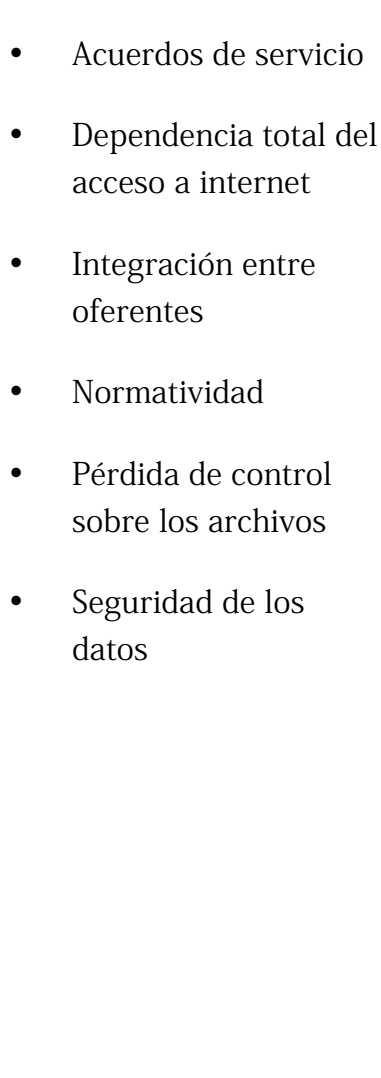 \\
\hline
\end{tabular}

A continuación se amplía la información sobre los pros y los contras de implementar cloud computing, de manera que el lector se pueda formar una mejor idea de esta tecnología.

Entre los puntos a favor del cloud computing se distinguen:

- Acceso universal a los archivos. Una de las grandes ventajas que tiene cloud computing sobre otros modelos de computación, es que el almacenamiento de los archivos se hacen en los espacios que tienen los usuarios en la nube, por tanto pueden acceder a ellos desde cualquier sitio que tenga conexión disponible a internet, permitiendo una mayor movilidad de los usuarios.
- Actualizaciones automáticas. Como en el cloud computing se paga por uso, las empresas siempre ofrecen las últimas versiones de sus programas garantizando el upgrade de versiones de forma natural. Tal como el servicio de correo de gmail el cual se va actualizando en forma continua y transparente para el usuario, o el caso de Force.com.

- Capacidad de almacenamiento y computacional ilimitada. La oferta de servicios del cloud computing en el modelo pago por uso, alquila recursos de acuerdo con las necesidades de la empresa, por el tiempo que se requiera. La ventaja es que el oferente, quien está especializado en estos servicios, tiene una capacidad disponible que puede satisfacer los requerimientos más exigentes de los clientes. Por ejemplo, el New York Times utilizó los servicios de amazon web services (CS2 y S3) para digitalizar en PDF once millones de artículos, para lo cual rentó cien computadores virtuales y cuatro Terabytes de disco duro. Esta tarea con los recursos propios del periódico hubiera tomado varios meses. Con el modelo cloud tomó un día y costo 240 dólares (Carr, 2008).

- Computación a bajo costo. Este es uno de los beneficios más importantes del cloud computing y uno de los argumentos más comunes para justificar el paso a este modelo. Varios estudios como los realizados por IDC para Force.com (Perry, Hatcher, et ál., 2009) se refiere a los ahorros que se pueden lograr por medio de cloud computing pueden ser de 3,1 a 3,9 millones de dólares, reducción en el TCO del 54\%, ROI de $721 \%$, respecto a la computación tradicional.

A continuación se presentan algunos estudios realizados por empresas consultoras, en los que se muestra que cloud computing es una buena opción financieramente.

Para iniciar, se presenta parte de los análisis financieros de realizado por el IDC en los gráficos 3 y 4 . 
Gráfico 3. Análisis de flujo de caja (Perry, Hatcher, et ál., 2009) Cash Flow Analysis
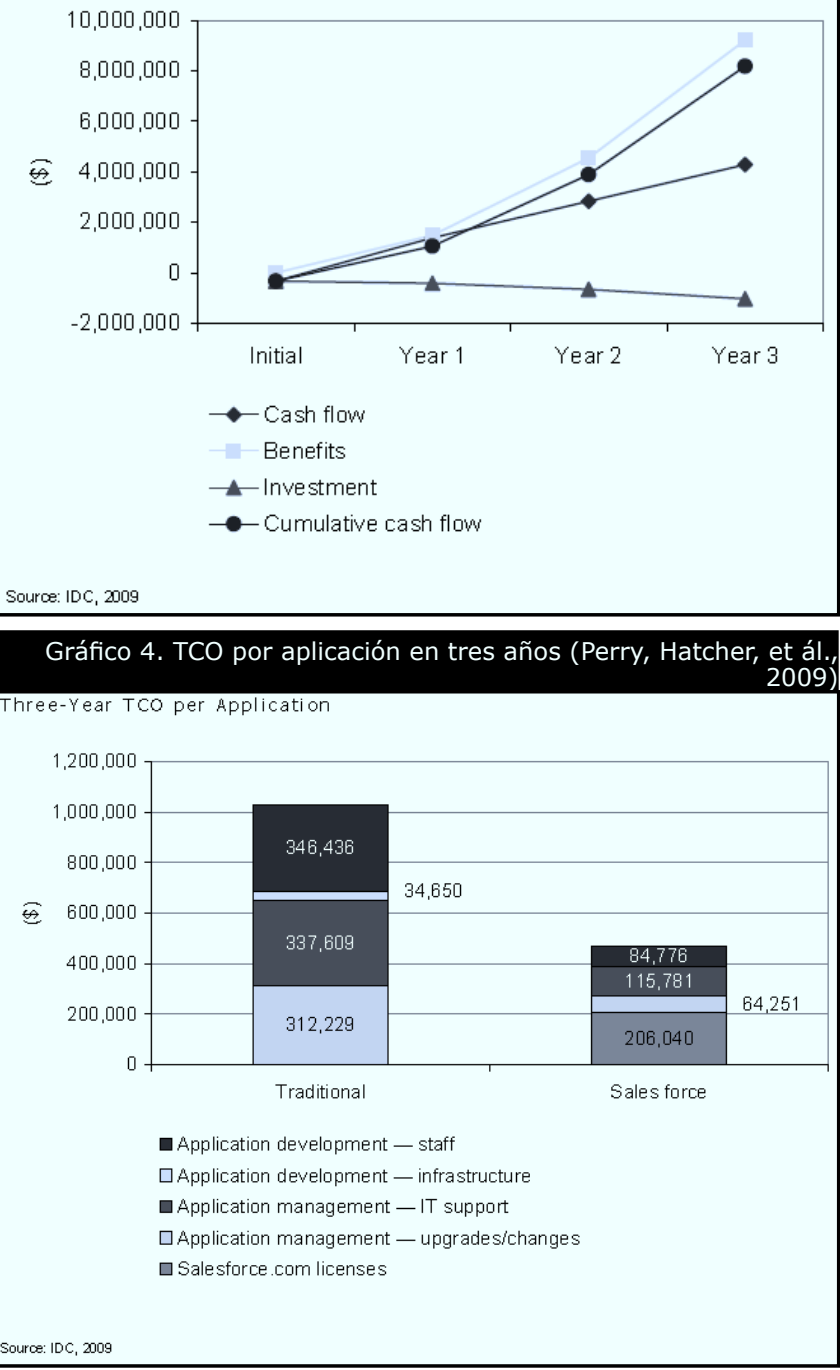

Como se puede ver la comparación es muy positiva para el cloud computing en el cual se aumenta el flujo de caja al no tener que hacer una inversión grande al principio con la compra de equipos ni programas y se puede tener más dinero disponible. El TCO disminuye pues como se paga por uso y los dueños de los equipos son los outsourcers, se paga solo el costo de mantenimiento.

Así mismo, en el anexo se muestra una comparación del TCO utilizando la calculadora de Windows azure ${ }^{1}$, que es la plataforma cloud de Microsoft, donde se obtiene un resultado similar al de IDC.

1 http://www.microsoft.com/windowsazure/tco/
El estudio de Forrester (Schadler, 2008) presenta los beneficios financieros de cloud computing y servicios basados en cloud.

Tabla 2. Beneficios financieros del cloud computing y los servicios basados en cloud

\begin{tabular}{|l|l|l|}
\hline Factor & On-Premise & Cloud \\
\hline $\begin{array}{l}\text { Tipo de } \\
\text { gasto }\end{array}$ & $\begin{array}{l}\text { Gastos de capital } \\
\text { (capex) } \\
\text { Gastos operativos } \\
\text { (opex) }\end{array}$ & $\begin{array}{l}\text { Gastos operativos } \\
\text { (opex) }\end{array}$ \\
\hline $\begin{array}{l}\text { Flujo de } \\
\text { caja }\end{array}$ & $\begin{array}{l}\text { Servidores y } \\
\text { software se } \\
\text { compran por } \\
\text { adelantado }\end{array}$ & $\begin{array}{l}\text { Los pagos se hacen } \\
\text { en la medida en que } \\
\text { se presta el servicio }\end{array}$ \\
\hline $\begin{array}{l}\text { Riesgo } \\
\text { financiero }\end{array}$ & $\begin{array}{l}\text { Todo el riesgo } \\
\text { financiero se toma } \\
\text { por adelantado } \\
\text { y con retorno } \\
\text { incierto }\end{array}$ & $\begin{array}{l}\text { El riesgo } \\
\text { financiero se toma } \\
\text { mensualmente y } \\
\text { se emparejan a los } \\
\text { retornos }\end{array}$ \\
\hline $\begin{array}{l}\text { Estado de } \\
\text { resultados }\end{array}$ & $\begin{array}{l}\text { Mantenimiento y } \\
\text { amortización de } \\
\text { gastos de capital }\end{array}$ & $\begin{array}{l}\text { Solo gastos de } \\
\text { mantenimiento }\end{array}$ \\
\hline Balance & $\begin{array}{l}\text { Software y } \\
\text { hardware so } \\
\text { contabilizan como } \\
\text { un activo a largo } \\
\text { plazo }\end{array}$ & $\begin{array}{l}\text { No aparece en el } \\
\text { balance }\end{array}$ \\
\hline
\end{tabular}

De la tabla 2 se resalta el cambio de gasto de capex a opex, en el que la inversión ya no se hace al principio afectando el capital (сарех), sino que se convierte en un costo operativo (opex), que se paga en la medida en que se utilice y, por tanto, el flujo de caja aumenta y no se tienen gastos de amortización de capital mejorando la salud financiera de la empresa y disminuyendo el riesgo.

West (2010) recopila los valores estimados de los ahorros obtenidos de acuerdo con varios estudios realizados. Por ejemplo, la oficina de presupuesto de Mark Forman ahorró entre un 90 y 99\% de costos operativos en IT, el informe de Ted Alford y Gwen Morton de Buzz Allen concluye que si las agencias gubernamentales se mueven a la nube pueden ahorrar entre 50 y $67 \%$. Un análisis de Merry Lynch dice que con esta tecnología se pueden hacer aplicaciones tres a cinco veces más baratas, lo que significa que pueden ahorrar entre 67 y 80\%, Rajen Sheth de proyectos de 
Google habla de un ahorro del 67\% al pasar el mail a plataforma cloud y Meritalk estima los ahorros totales en $39 \%$.

Este mismo autor plantea que dada la variación en las cifras de los ahorros, muesta que todavía existe cierta incertidumbre en los ahorros de costos debido a la cantidad de factores que entran en la estimación de los costos.

Para terminar este punto, de acuerdo con el estudio Clearing the clouds de McKinsey, cloud computing es una buena alternativa económica para empresas pequeñas y medianas pero no para grandes empresas, esta idea también es reforzada por (Lohr, 2001).

Si se quiere tener una idea de cuánto puede valer una solución cloud puede ver un estimado con las calculadoras de amazon ${ }^{2}$ o de Windows azure.

- Disminución en el tiempo de desarrollo y atención a usuarios: otra de las ventajas más publicitadas por los oferentes es la reducción del tiempo dedicado al desarrollo de aplicaciones (Perry, Hatcher, et ál., 2009) habla de reducciones entre 76 y $85 \%$ en el tiempo de desarrollo y del $37 \%$ en servicio al cliente. + Publicidad \& marketing (2010) se refiere a una reducción del $98 \%$ de requerimientos a soporte técnico, lo que permite que el personal de TI se pueda dedicar a otras labores dentro del área (Clarck, 2010) hace mención a los ahorros entre 67 y $85 \%$ en desarrollos iniciales.

Narasimhan (2009) hace mención a incrementos de 2 y 3 veces para sacar productos al mercado utilizando cloud y otro ejemplo de un producto que normalmente demora tres años y lo sacaron en seis meses, lo que dio como resultado reducciones entre 50 y $75 \%$ en el tiempo.

Estas reducciones se deben principalmente a que son soluciones que ya están trabajando y la empresa comienza a utilizarlas apenas se paga la suscripción, por esto en algunos casos la implementación de soluciones cloud se hace en un solo día.

- Escalabilidad. Este beneficio del cloud computing permite crecer en la infraestructura disponible para la empresa en poco tiempo y bajos costos.

2 http://calculator.s3.amazonaws.com/calc5.html
Clarck (2010) plantea una comparación en costos de escalamiento por adquisición de la empresa Vs. escalamiento por cloud (gráfico 5). Cabe aclarar que con el modelo cloud de pago por uso, cuando se deje de necesitar tanta infraestructura, simplemente se deja de pagar, en cambio si se comprara en la empresa, cuando bajen los requerimientos, los equipos quedan subaprovechados en la empresa, esto refuerza el concepto de cambio entre capex y opex.

Como ejemplo, la empresa Animoto paso de 50 instancias en amazon a 3.500 en tres días, lo que muestra la importancia de la posibilidad de escalar con un pico de información, que luego se puede devolver o dejar de pagar cuando pase el pico.

- Independencia de software y máquinas. Uno de los componentes del cloud computing es el SaaS o software "as a service", en donde se ofrecen los programas que necesite la empresa en modalidad de pago por uso y acceso vía web.

Esta ventaja permite que el trabajo sea centrado en el usuario y en el documento, mas no en el equipo en el que tenga instalados los programas, permitiendo movilidad por parte de los empleados y compartir información entre ellos mejorando la productividad entre los empleados. Esto se puede ver con la suite de google docs por ejemplo.

Esta independencia refuerza la viabilidad financiera del cambio, pues ya no se tiene que hacer la inversión por adelantado cargándola al balance, sino que se convierte en el pago de un servicio.

Los puntos en contra se mencionan en la mayoría de artículos consultados como asuntos por mejorar, por lo que se hace la aclaración al lector interesado en consultar bibliografía adicional, ya que estos puntos a pesar de ser pocos pesan bastante en el momento de tomar la decisión de implementar una solución cloud computing o no.

- Acuerdos de servicio. Los niveles de prestación del servicio (o Service Level Agreements, SLA) se refieren a la disponibilidad que debe tener el servicio contratado para la empresa. En el 


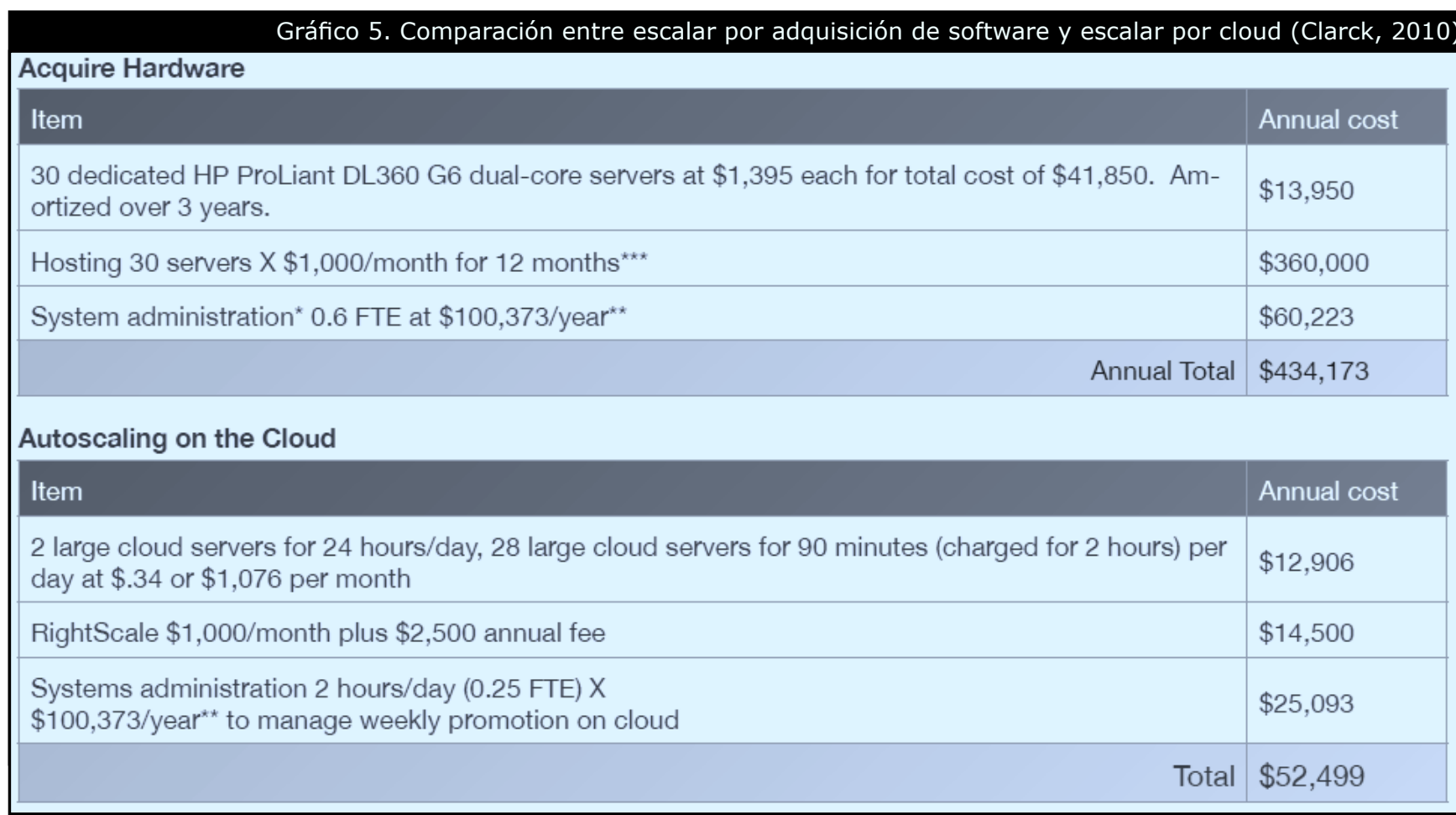

caso de cloud computing por lo general se contrata un $99,9 \%$, pero no siempre se llega a esto por diferentes razones. El impacto de las fallas en el servicio pueden ser nefastas para la empresa, por ejemplo (Zhen, 2008) cita el ejemplo de Amazon quien

encontró que por cada 100 milisegundos de latencia hay un costo del $1 \%$ de sus ventas. Google ha encontrado que por cada 0.5 segundos extra en el tiempo de búsqueda de páginas, el tráfico disminuye en un $20 \%$. Un corredor podría perder $\$ 4$ millones en ingresos por milisegundo si su plataforma de comercio electrónico es de 5 millisegundos más que la competencia.

Lo que muestra que una variación en el cumplimiento del servicio puede significar pérdidas para la empresa.

Por ser un modelo que está en su etapa de maduración, no hay todavía estandarización para los LSA, que de acuerdo con la opinión de los expertos se irá dando con el tiempo.

- Dependencia del acceso a internet. Este es el mayor inconveniente que se le encuentra al modelo cloud en países en vía de desarrollo, pues cloud depende totalmente de la conexión a internet para que funcione, así se hayan sacado versiones offline como mail Google, pues se necesita la conexión para sincronizarse. Y no solo requiere conexión sino buena velocidad para que los LSA puedan cumplirse, así que si no se tiene una buena conexión a internet con un precio competitivo, cloud no sería una alternativa viable.

- Integración entre oferentes y administración de aplicaciones. Cada uno de los proveedores generan sus estándares y sus formas de trabajo. Si bien todas son basadas en web como tecnología común, ofreciendo servicios de alta calidad, se pueden presentar problemas para conectar un servicio con el otro, para esto se están trabajando en alianzas e interfaces entre empresas por ejemplo el CRM Salesforce con Google Docs, así como formas de comunicación a través de webservices.

Adicional a los problemas de comunicación entre programas Zhen (2008) plantea dificultades en la administración de estas, presentando como ejemplo el escalamiento de la infraestructura, que inicialmente debería ser automática en la medida en que el cliente la requiera y debe ser realizada por el administrador del sistema. Por tanto si no se tienen formas de administrar las aplicaciones y servicios, se pueden presentar problemas en la prestación del servicio, teniendo consecuencias 
directas en la disponibilidad de la información o de las aplicaciones para los clientes con consecuencias directas sobre las actividades de las empresas o en sus utilidades.

- Normatividad. En la actualidad el cloud computing es una tecnología que está pasando de su iniciación a la madurez, pero todavía se tienen diferentes empresas que crean sus propios estándares, lo que impide realizar certificaciones bajo una norma específica, así como facilitar la interoperabilidad entre los diferentes servicios que se ofrezcan. De todos modos hay estándares para el intercambio de datos por medio de web services (basado en SOA) habilitaría puentes para el intercambio de datos.

- Pérdida del control sobre los archivos. Uno de los grandes peros que se tienen para el cloud computing es que la empresa cede el control de la información, como plantea el creador del GNU Richad Stallman (Johnson, 2008). Se genera una desconfianza del control de la información a un tercero que la alojara en sus servidores, los cuales están por fuera de la empresa. Dada la importancia de la información para las organizaciones, se tiene más tranquilidad cuando la información está al interior de la empresa, pero como argumento para este factor se tiene que las empresas oferentes de cloud computing tienen el personal adecuado y los recursos necesarios para atender cualquier eventualidad en sus equipos, por tanto su información será manejada por un área de tecnología que para tenerla dentro de la empresa sería demasiado costoso.

- Seguridad de los datos. Este factor es común en todos los autores consultados, pues al ser aplicaciones basadas totalmente en web la seguridad de los datos es una preocupación constante debido a los ataques de hackers que puedan vulnerar la seguridad del sistema. Para este aspecto se puede argumentar por medio del siguiente ejemplo: ¿Cuántas empresas pueden tener más personal experto y más recursos computacionales que Google para proteger la seguridad de los datos? Con esto lo que se quiere mostrar que las empresas oferentes tienen un mejor soporte para la seguridad de los datos que lo que puede tener una empresa por medio de firewalls y otras opciones

Un documento realizado por Garnet group, citado por Beooklin (2008), plantea que los aspectos en seguridad que se deben tener en cuenta para seleccionar proveedores de cloud computing son los siguientes: acceso privilegiado de usuarios, cumplimiento de normatividades, ubicación de los datos, segregación de datos, recuperación de datos, apoyo a la investigación y viabilidad a largo plazo.

Existen detractores del cloud computing que muestran sus debilidades, entre estos se encuentran Richard Stallman creador de la fundación de software libre, quien dice que el cloud computing es una trampa y lo califica como una gran campaña de marketing, pero su razón principal para no utilizar cloud computing es que se pierde el control (Johnson, 2008). En una revisión de publicaciones especializadas en temas de tecnología, para Network World (2008) entre los retos del cloud computing se encuentran: privacidad de los datos, seguridad, licencias de software, aplicaciones, interoperabilidad, cumplimiento normativo, SLA que garanticen un determinado rendimiento, monitorización de red.

\section{Empresas más importantes}

En el mercado que se está desarrollando para cloud computing es importante revisar quienes están ingresando en este; aquí se puede ver que grandes empresas que han sido pioneras en los servicios de internet están haciendo sus proyectos cloud, apostándole al crecimiento y estabilización de este mercado. Así mismo se pueden observar empresas tradicionales como Microsoft que por lo general llega después que los demás, pero que su presencia asegura que es un mercado con potencial. De acuerdo con los diferentes componentes del cloud computing se listan a los proveedores para dar un mejor panorama de los actores principales. 
A. Infrastructure as a Service (IaaS)

- Amazon Web Services

- VMWare

- Elastra

- $\quad 3$ Tera

- Xen

- XCalibre

- Nirvanix

- $\quad$ EngineYard

- Joyent

\section{B. Platform as a Service (Paas)}

- Mosso

- Google App Engine

- SalesForce

- Morph

- Heroku.

\section{Software as a Service (SaaS)}

- Google docs

- Salesforce

- Zoho

- Windows azure

- Mobile me.

A modo de síntesis los factores comunes que se deben tener en cuenta para la implementación del cloud computing en una empresa nueva son:

- Calidad del servicio de internet

- Costo

- Capacidad computacional a corto y largo plazos
- Escalabilidad

- Acuerdos de servicio

- Seguridad

- Interoperabilidad

- Administración

- Monitoreo

- Auditoría.

\section{El emprendimiento en Colombia}

Sobre el emprendimiento en Colombia se hace una breve introducción de cómo funciona el sistema que está siendo liderado por el SENA y ver cuáles son las iniciativas que están apoyando para tener una mejor comprensión de las empresas y sus características. Cabe anotar que las iniciativas del SENA cuentan con un apoyo decidido por parte del gobierno nacional por medio de Colciencias y los ministerios, creando el sistema nacional de innovación, desarrollo tecnológico y emprendimiento en Colombia.

El documento Conpes (Consejo Nacional de Política Económica y Social), instancia coordinada por el Departamento Nacional de Planeación (DNP) "Consolidación del sistema nacional de innovación, desarrollo tecnológico y emprendimiento en Colombia" plantea, entre otros aspectos, los actores del sistema y los instrumentos para su implementación (tabla 3). En este documento se puede apreciar si se tiene un apoyo decido del los estamentos gubernamentales como los ministerios y el SENA y su interacción con las universidades y los gremios (universidad-empresaEstado) se dan todas las condiciones para que las personas hagan realidad su idea emprendedora bien sea por medio del apoyo gremial, de su universidad o por medio de las incubadoras de empresas y los centros de desarrollo tecnológico. En esta misma tabla 3 se muestran los instrumentos por medio de los cuales se puede implementar el sistema, en el que se subraya la formación técnica y tecnológica de la población. 
Tabla 3. Sistema nacional de innovación en Colombia

(Conpes, 2005)

\section{Instrumentos para,la.implementación del sistema} deinnovación

Actores del sistema

- Cadenas productivas

- Red de parques de nuevas tecnologías

- Incubadoras de empresas y centros de desarrollo tecnológico

- Cadenas de formación del SENA articuladas con la educación media y las universidades

- Centros de investigación aplicada y de desarrollo tecnológico de las universidades

- Clusters de conocimiento

- Consejo Nacional de Innovación, Desarrollo

Tecnológico y Emprendimiento, con Secretaría Técnica ejercida por el SENA.

- Política nacional de largo plazo para el desarrollo del sistema

- Reducción de las fallas en el mercado de capitales (fondos: venture capital, cuasi capital y garantías)

- Fondos de fomento y beneficios a la inversión

- Apropiación de tecnología desarrollada en el mundo

- Alianzas y convenios

- Interacción de colectivos empresariales para la innovación desde el sector productivo y el mercado

- Formación técnica y tecnológica para la innovación, el desarrollo tecnológico y el emprendimiento

Además el documento Conpes 3582 plantea como estrategia complementaria el "fomentar la utilización de las tecnologías de información y comunicaciones (TIC)" (Conpes, 2009), confirmando la importancia que tiene el tema de las TIC en cuestiones de emprendimiento.

El SENA es la institución oficial encargada de liderar el tema de emprendimiento en Colombia y en su documento (SENA, 2009), se hace presente la orientación hacia la tecnología, no solo en el desarrollo de hardware o software, sino también en la transferencia de tecnología (gráfico 6).

Barboza Flores (2008) expone los tipos de incubadoras "en función del problema que se quiere atender, de los recursos disponibles y/o de las habilidades, fortalezas u oportunidades que se desean aprovechar". La clasificación propuesta por este autor es la siguiente:

- Especializadas. Orientadas a una rama o actividadeconómica oaunáreadelconocimiento,
- Cadenas productivas, redes empresariales y gremios de la producción y los servicios

- Comunidades organizadas y sectores solidarios

- Sistemas de financiación

- SENA: presencia efectiva en todo el territorio nacional y relación directa y oportuna con las cadenas productivas regionales

- Ministerio de Hacienda y Crédito Público

- Dirección de Impuestos y Aduanas Nacionales (DIAN)

- Ministerio de la Protección Social

- Ministerio de Educación

- Ministerio de Comercio, Industria y Turismo

- Ministerio de Comunicaciones

- Ministerio de Agricultura y Desarrollo Rural

- Otros ministerios

- Incubadoras de empresas de base tecnológica

- Centros de desarrollo tecnológico

- Mesas sectoriales

- Universidades y unidades de emprendimiento

- Departamento Nacional de Planeación

- Fuerzas Militares de Colombia

- Colciencias

- Icetex

\section{Gráfico 6. Enfoque en el emprendimiento colombiano (SENA, 2009)}

"SENA: Una Organización de Conocimiento" - El Nuevo Enfoque

\section{Líneas de Acción}

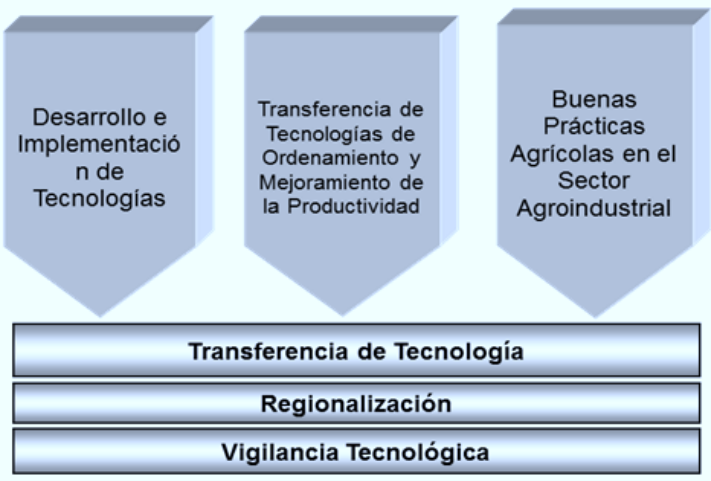


por ejemplo, alimentos, biotecnología, ingeniería de software, artesanía, etcétera.

- Multipropósito. Albergan empresas de diversas actividades económicas y tipos de productos y servicios.

- Alta tecnología o base tecnológica. Se especializa en empresas nuevas cuyo negocio está basado en procesos, productos o servicios donde la tecnología es nueva o innovadora, considerada como tecnología de punta y donde el conocimiento se convierte en el elemento que aporta un alto valor agregado y es el que permite mantener ventaja competitiva.

- Virtual. También conocida como incubación extramuros y en la que no se dispone de instalaciones físicas a ser ocupadas por los emprendedores, sino que el paquete de servicio puede ser accesado sin estar físicamente en la sede de la incubadora

A partir de esta clasificación nos enfocaremos en las empresas de base tecnológica por la afinidad que tienen con los temas relacionados con la tecnología y se parte de un mejor nivel de aceptación de nuevas tecnologías, que podría ser un obstáculo en otras empresas que no tienen conocimientos sobre TIC.

\section{Resultados de la investigación}

Teniendo en cuenta los factores resultantes del análisis del marco teórico, se realiza una encuesta para validar que estas tendencias en mercados más evolucionados que el colombiano, también se presentaban en nuestros emprendedores. Para esto se define entonces como población a personas con empresas nuevas y que tengan conocimiento o estén relacionados con temas de tecnología. Luego de analizar la información disponible del sistema nacional de innovación, se encuentra que los proyectos que se encuentran en incubadoras de base tecnológica se ajustan a las características de esa población objetivo. Inicialmente se planteaba un muestreo aleatorio en empresas de base tecnológica, pero gracias a la concentración de la población objetivo en la incubadora del SENA, se decide contactar a los proyectos presentes, de los cuales cincuenta de estos respondieron. Por medio de una encuesta semiestructurada, se realizan preguntas para caracterizar las empresas de base tecnológica respecto a su objeto del negocio, tamaño de la empresa (cantidad de empleados), capital inicial y porcentaje de este capital dedicado a sistemas de información, importancia de TIC en la empresa y validación de los factores planteados en la sección anterior.

Los resultados se presentan a continuación:

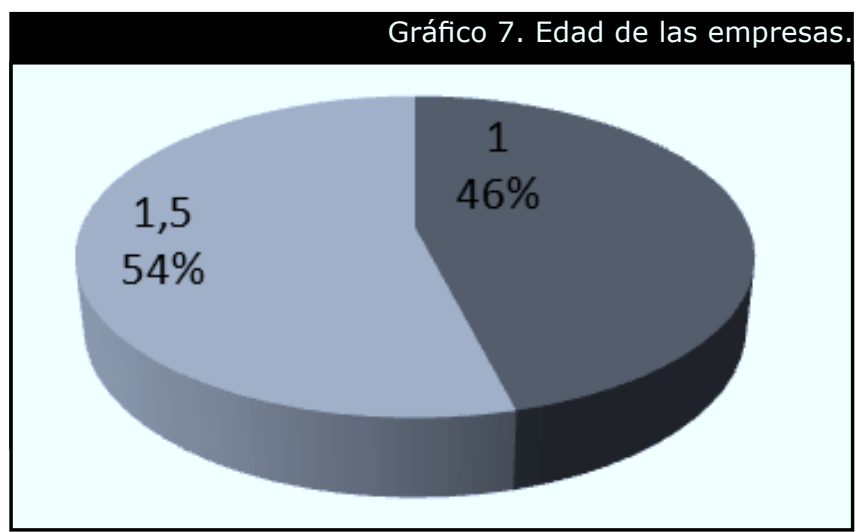

Los proyectos tienen entre 1 y 1.5 años, esto se debe a las reglas de la incubadora en donde se alojan en las instalaciones del SENA durante la fase de incubación (gráfico 7).

Entre los objetos del negocio, se tienen iniciativas diferentes que pueden resumirse en los siguientes campos (gráfico 8):

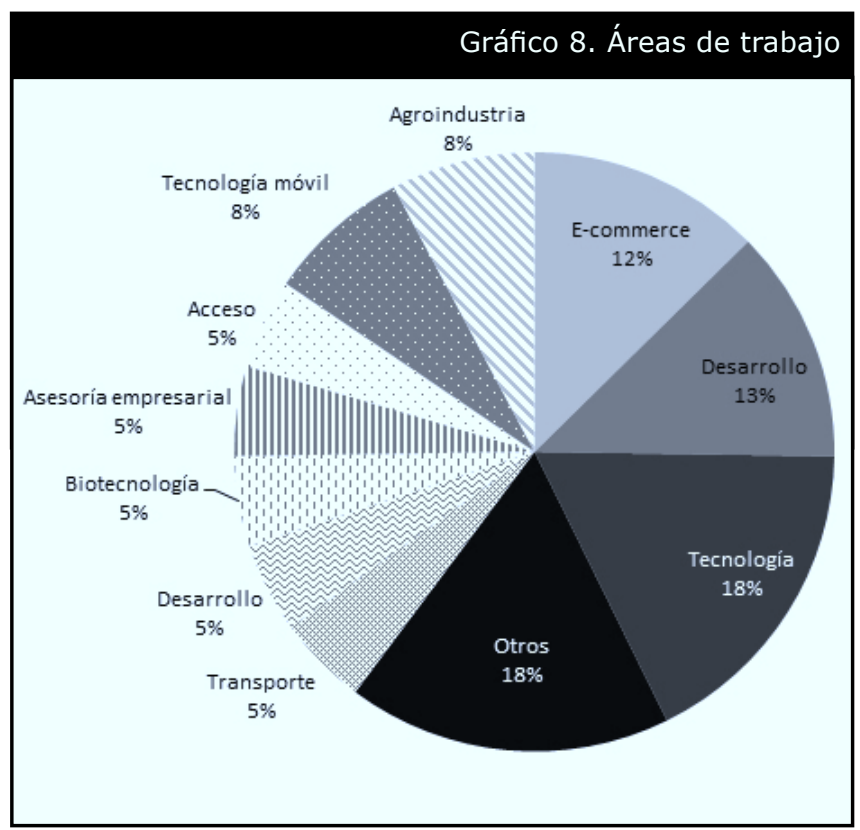

Se puede ver la fuerte tendencia a enfocarse en productos de computación (42\% representado en empresas dedicadas a tecnología, desarrollo de software o desarrollo web, bases de datos y comunicación).

La cantidad de personas que integran la empresa es pequeña, se tiene una minoría de empresas unipersonales, pues para estos proyectos se requiere un equipo interdisciplinario, que permita complementar los saberes de cada integrante (gráfico 9). Esos tamaños comprueban lo dicho por Camacho Pico (1988) sobre 
Gráfico 9. Cantidad de personas por empresa

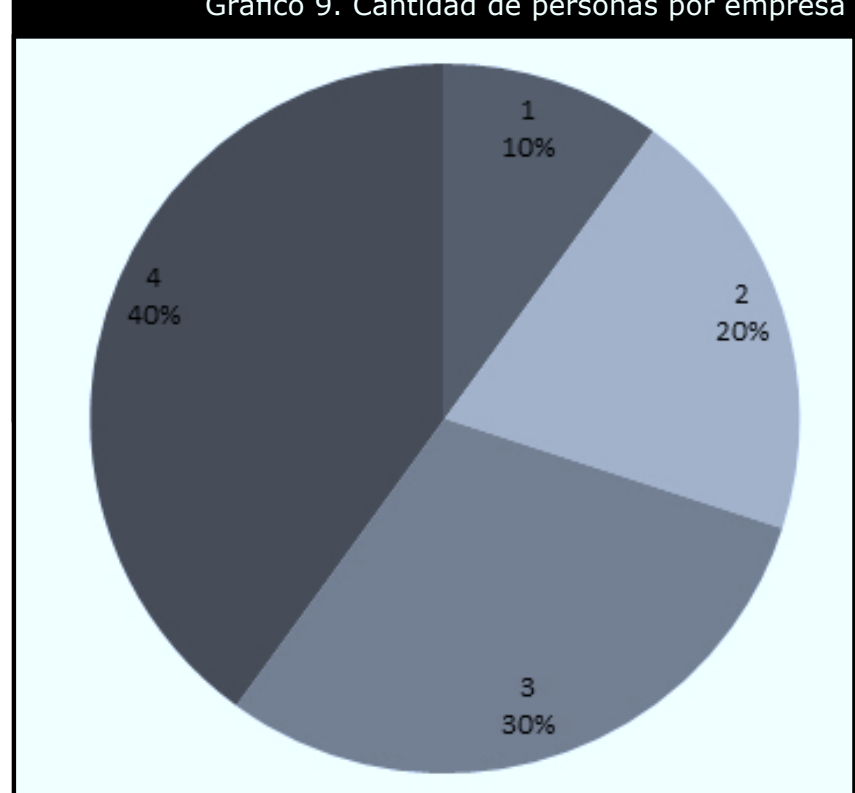

el tamaño de las empresas de base tecnológica, cuando sostiene que una de las características de estas empresas es el tamaño reducido de sus integrantes.

El capital inicial de la empresa tiene un promedio de 19.680.000 de pesos y una desviación estándar de 6.254.507 de pesos. Su mediana es de 21.500.000 de pesos, lo que muestra que el capital para iniciar un proyecto de emprendimiento de base tecnológica está cercano a los 21 millones de pesos, siendo esta una cantidad de dinero alta para las condiciones del país, por lo que es necesario identificar en qué puntos se pueden realizar ahorros para no tener que invertir tanto.

Gráfico 10. Comparte recursos tecnológicos

¿Estaría dispuesto a compartir recursos tecnológicos?

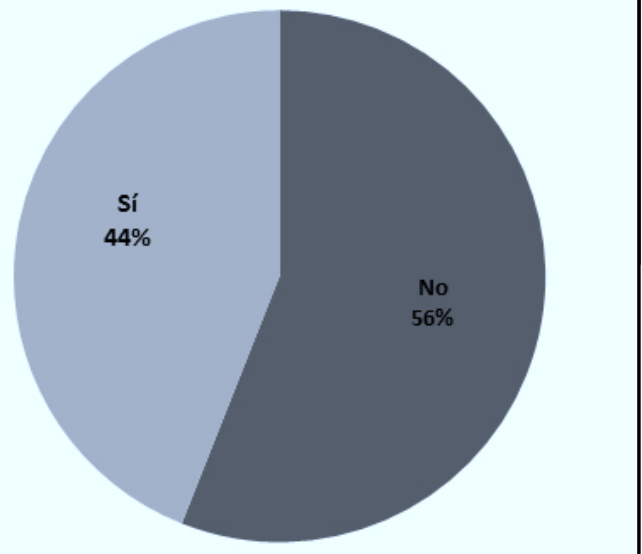

El porcentaje del capital inicial destinado a tecnología es de $49,4 \%$ en promedio, con una desviación estándar del $11 \%$, lo que nos permite expresar que los ahorros logrados en tecnología tendrán un alto impacto en la formación de nuevas empresas y el capital que se destina a la adquisición de tecnología se puede concentrar en otros aspectos que pueden ser fundamentales para la empresa o que ayuden a cumplir con su misión de una forma directo

Tabla 4. Importancia de las TIC de acuerdo con el porcentaje de capital invertido en ellas

\begin{tabular}{|c|c|c|c|c|c|c|c|}
\hline \multicolumn{7}{|c|}{ \% del capital inicial dedicado a TIC } \\
\hline Importancia \\
\hline 1
\end{tabular}

En la tabla 4 se destaca el caso de un 56\% de la muestra, que considera que la tecnología tiene una importancia entre baja y media ( 1 a 3 ), pero de estas empresas, el $64 \%$ de ellas tienen una inversión en tecnología que supera el 50\%, lo que evidencia que son empresas en donde la tecnología no representa lo más importante para la empresa, pero que en términos financieros su importancia es notoria, al haber destinado una gran parte de su presupuesto en ella; por tanto si para estas empresas las TIC no son tan importantes, no deberían gastar tanto dinero en ellos y se deberían ofrecer alternativas que permitan cumplir con ello. El gráfico 10 ilustra que la gente si bien no está del todo en desacuerdo sobre compartir recursos tecnológicos de hardware y software (uno de los principios en los cuales se basa el cloud computing), todavía falta un camino por recorrer para que esta idea sea aceptada. 
Se les pregunta si saben de qué se trata el cloud computing y el 50\% afirma tener conocimiento, lo que permite formular que los emprendedores que conocen del tema pueden tener una mayor aceptación sobre tecnologías cloud y quienes no conocen sobre esta tecnología pueden presentar cierta resistencia por desconocimiento (gráfico 11).

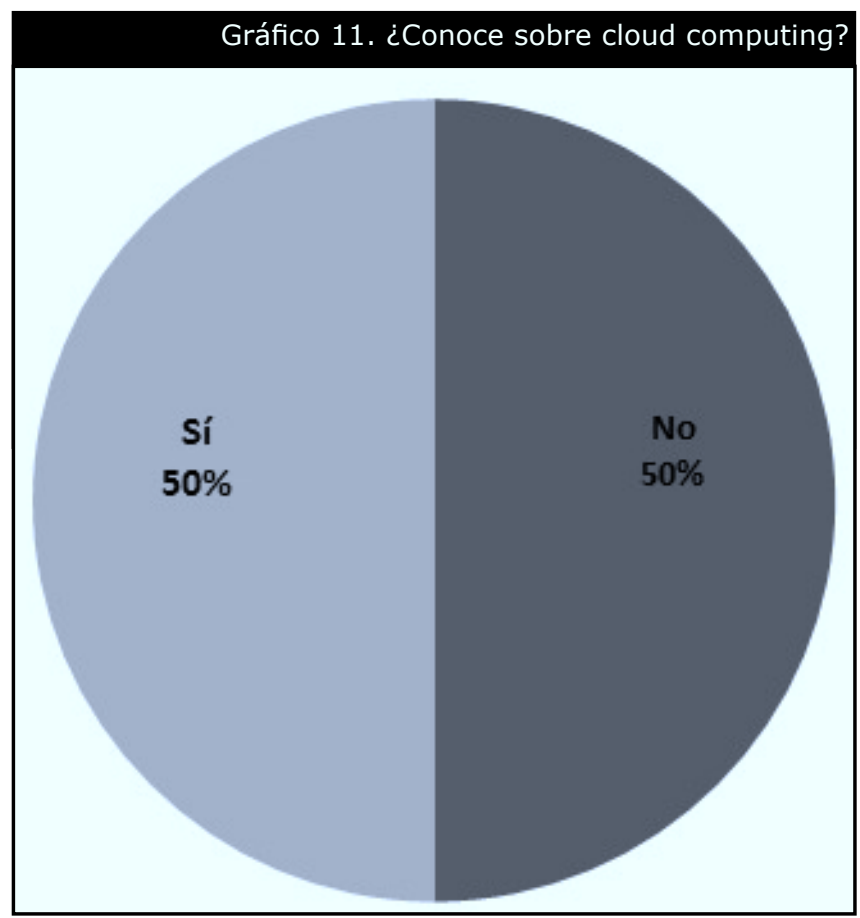

El gráfico 12 condensa la importancia que los encuestados le dan a los beneficios más representativos que ofrece el cloud computing. Vale resaltar que el comportamiento de los encuestados difiere en algunos puntos sobre la aceptación de las características del cloud computing, en el que la accesibilidad de la información desde cualquier sitio con conexión a internet, que es una de las grandes ventajas y una de las razones por las cuales se está imponiendo el cloud computing en el mundo, el $48 \%$ de los encuestados le asignan una importancia baja (entre 1 y 2).

La reducción en los costos de software es calificada por el $54 \%$ de los encuestados, con una importancia media baja (entre 2 y 3), lo que indica que para ellos los gastos en software no tiene mucha importancia; esta concepción respecto al costo puede ser debido a la costumbre del alto valor por la implementación de sistemas de información en las mismas empresas y que

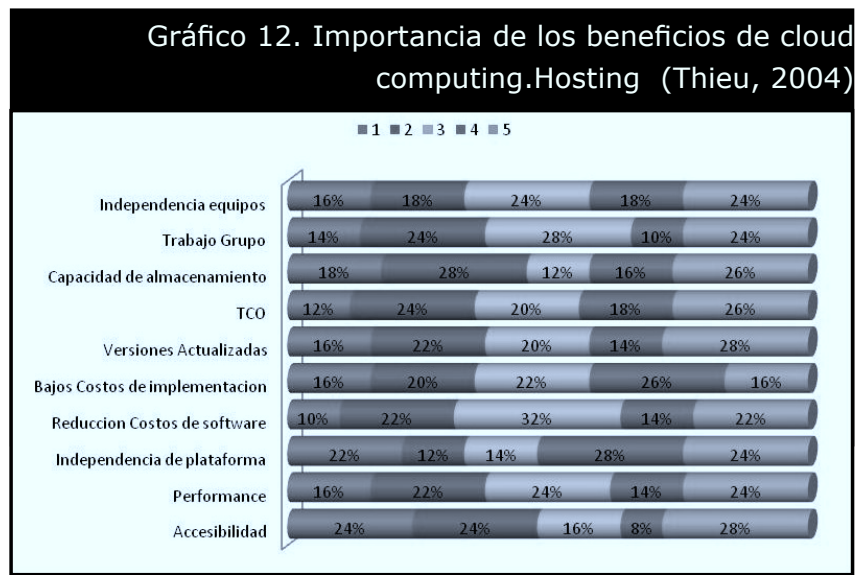

por lo general son estándares en el mercado; por tanto si no se pueden cambiar, pues su importancia radica en conseguir los recursos financieros para poder pagarlos. Además se plantea, que la falta de importancia se debe al desconocimiento de opciones para disminuir costos, por lo que sería importante hacer mayor difusión de esta tecnología.

Así mismo, el trabajo en grupo y el performance computacional tienen una importancia media baja (23). La explicación para que el trabajo colaborativo no tenga tanta importancia es porque hasta el momento no es una práctica muy extendida entre empresas pequeñas, debido entre otras cosas a la falta de conocimiento de las herramientas que habiliten esta opción. El performance de los equipos sorprende que se considere con esta importancia, pues nos estaría diciendo que los emprendedores consideran que no necesitan tanta capacidad computacional, aunque también podría deberse a que compran lo que necesitan al principio sin tener en cuenta como puedan crecer a futuro.

Los costos de implementación y mantenimiento tienen una importancia medio-alta (3-4), lo que muestra que los encuestados son conscientes del costo de los sistemas de información, pero no es tan importante para ellos, posiblemente porque no conocen otras alternativas que permitan disminuir los costos y evaluar el dinero invertido.

El costo total de propiedad (Total Cost of Ownership) es una medida que permite valorar los costos y los beneficios relacionados con la inversión en tecnologías de la información, utilizada por muchas empresas que trabajan en tecnología, pero que no está del todo 
posicionada dentro de la parte financiera por lo que al momento de ver el comportamiento financiero de la empresa, los equipos se toman como un gasto necesario; puede ser por esto que las opiniones sean variadas en términos de importancia (44\% lo califican con importancia alta y otro porcentaje igual lo califican con importancia media baja), sin que se destaque uno, por lo que habría que popularizar este indicador entre las empresas que inviertan en tecnología para que sean conscientes de su importancia.

Así mismo la independencia de plataformas tiene una percepción de importancia similar en cada nivel, lo que muestra que si bien se puede considerar importante no se puede generalizar para todos los grupos. En las tendencias mundiales estos aspectos son muy llamativos para los usuarios, quienes pueden manejar sus datos desde donde quieran sin tener que estar atados a una plataforma o a un programa específico que solo está en un su lugar de trabajo. Se plantea como explicación que hasta el momento la gente tiene asignados sus equipos en las empresas y la posibilidad del teletrabajo todavía está por desarrollar, por tanto no se tiene tanta necesidad de abrir los archivos en otros sitios diferentes a los acostumbrados (casa y trabajo); además el liderazgo de programas bajo plataforma Windows, permite que la mayoría de archivos se puedan leer en casi cualquier computador

La independencia de los equipos de cómputo, las versiones actualizadas y el Tco, tiene una percepción de importancia alta. El Tco y el hecho de estar actualizado con las versiones comparten las mayorías con otros niveles, posiblemente a que hay personas que son conocedoras del tema y lo califiquen con un nivel y otros que no tengan tanto conocimiento y le asignen otro valor. La independencia de equipos de cómputo es calificada por un $52 \%$ como alta, posiblemente a que los encuestados no quieren sentirse atados a una máquina específica o a su lugar de trabajo y si existe alguna alternativa que les permita esto será bienvenida.

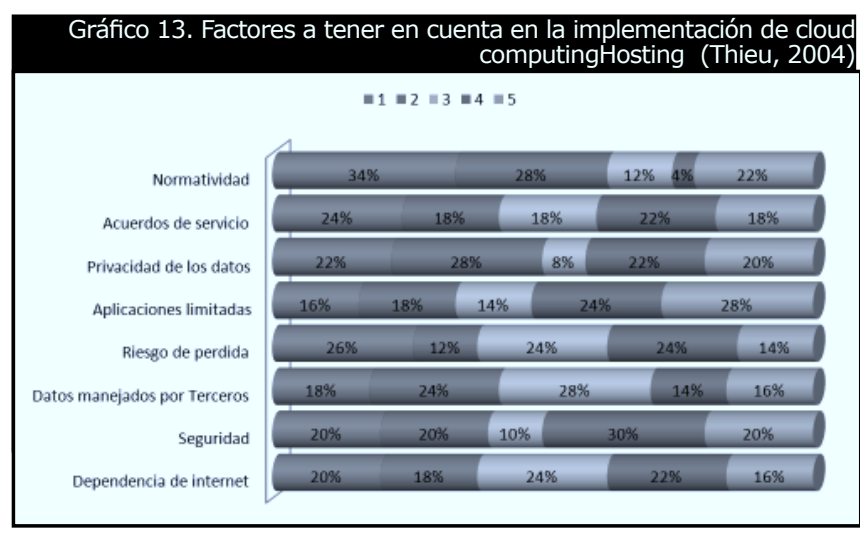

En el gráfico 13 se observa que la privacidad de los datos, que es una de las mayores preocupaciones en el concierto mundial, no tiene una importancia baja (12) para los encuestados, hecho que sorprende pues en la pregunta de si compartiría información, la mayoría dijo que no. Así mismo a los acuerdos de servicio y a la normatividad tampoco se les asigna tanta importancia, posiblemente porque no se tiene mucho conocimiento sobre el impacto de estos temas en la implementación de nuevas tecnologías.

Que los datos sean manejados por un tercero tiene una importancia medio-baja (2-3), lo que estaría más o menos de acuerdo con la opinión en la pregunta con respecto a compartir información. Esto abre una puerta para iniciativas públicas o privadas que desarrollen infraestructuras cloud, para el uso intensivo de varias empresas o proyectos y que por economías de escala se puedan tener mejores precios o si es por una entidad pública, al lograr un buen acuerdo se podría incluir dentro de las incubadoras aplicaciones sobre cloud para el funcionamiento de una empresa.

La dependencia de internet y el riesgo de pérdida de la información tienen una importancia medio-alta (3-4). La primera es una preocupación constante en países en los que el cubrimiento de internet y la velocidad disponible no son las mejores, ya que las aplicaciones cloud sí requieren una velocidad mínima para poder funcionar de manera adecuada; en Colombia esta situación es menos grave cada vez, pues los niveles de servicio en estos aspectos se están mejorando continuamente. El tema de recuperación de datos, se toma en este nivel por la importancia de la información para cada empresa y siempre va a ser un motivo de preocupación la parte de copias de seguridad y recuperación de información perdida; pero si se contratan servicios con empresas dedicadas a estos aspectos tendrán mayores y mejores recursos a los de una empresa que no se dedique a los servicios tecnológicos, permitiendo descargar así esa responsabilidad en los terceros.

La seguridad y las aplicaciones limitadas son los temas de mayor importancia para los encuestados, y se debe precisar cuáles son los temas más importantes en los cuales deben trabajar los oferentes de servicios cloud, para poder llegar a un determinado mercado, pues si las soluciones ofrecidas no dan la sensación de ser seguras, nadie pasará de su sistema in house a un sistema externo y de acceso web. Es claro que no hay ningún sistema seguro, pues existen como mínimo debilidades por parte de los usuarios en términos de seguridad. Además la oferta limitada de aplicaciones cloud puede preocupar a los usuarios, ya que no estarían seguros de 
si existe una aplicación que se ajuste a sus necesidades o exigencias, pero esto está cambiando a través del tiempo, ya que los grandes desarrolladores de software están ofreciendo aplicaciones sobre cloud.

\section{Conclusiones}

El cloud computing es la tendencia que se impondrá en un futuro para los sistemas de información empresariales, lo cual se puede verificar por medio de la variedad de aplicaciones existentes y los jugadores que entraron en el mercado, como Google, Microsoft, IBM, Oracle, entre otros, quienes tienen soluciones de diferentes rangos de precios incluyendo aplicaciones gratuitas como Google docs, que pueden satisfacer las necesidades de información de los proyectos emprendedores.

Sus características de bajo costo y facilidad de implementación y uso, convierten al cloud computing en una alternativa que apoya las iniciativas de emprendimiento al permitirle a los integrantes concentrarse en el core del negocio sin que los sistemas de información se conviertan en un problema de tiempo, conocimiento o dinero.

Acerca de la inversión en tecnologías de la información Spy (2010) utiliza la frase "10 millones es el nuevo 100 millones de pesos”, para indicar que la inversión necesaria en cuanto a iniciar un negocio mediante la utilización de cloud computing es menor a los costos para iniciarlo antes de esta tecnología, mostrando que en efecto es un beneficio que puede apoyar la creación de empresas.

Entre los aspectos a tener en cuenta en el momento de implementar aplicaciones cloud se debe cuidar quien maneja los datos, cuáles estándares y normatividad está vigente o cumple el oferente, si existe interoperabilidad en los sistemas, la administración de las aplicaciones y los niveles de servicio la seguridad.

Para los emprendedores de proyectos de base tecnológica, el cloud computing no es un concepto desconocido del todo, y a pesar de tener varias opiniones similares con el resto del mundo, hay algunos aspectos en los cuales su opinión es diferente. Entre las ventajas que ofrece el cloud, para los encuestados, es más importante la independencia de plataformas y programas, así como tener versiones actualizadas de las aplicaciones que utilicen y menos importantes la posibilidad de acceder a la información desde cualquier parte y los costos del software, siendo este último uno de los comportamientos que difieren del contexto internacional, debido posiblemente a los costos de implementación y el desconocimiento de alternativas. De ser así el cloud computing tiene una gran aplicabilidad, debido que la oferta creciente en cuanto a aplicaciones permite que el mercado ofrezca mejores precios debido a la competencia, lo que redunda en beneficio del usuario.

Respecto a los aspectos a cuidar, los usuarios coinciden en que la seguridad y la cantidad de aplicaciones disponibles en el mercado son los factores más importantes en el momento de considerar soluciones cloud coincidiendo con los autores revisados y considerando que el hecho de que sus datos sean manejados por terceros como los menos importantes, lo cual muestra una evolución en el pensamiento de los usuarios. Esto coincide con lo planteado por varios expertos en el tema, en el que las empresas dedicadas a ofrecer los servicios cloud tienen más recursos especializados dedicados a una correcta gestión de la información a lo que pueden tener unas empresas medianas o pequeñas.

Como conclusión, el cloud computing es una opción válida para apoyar proyectos de emprendimiento, en los que a partir de una correcta asesoría para la selección de las aplicaciones y servicios que necesiten los proyectos, gran parte de los recursos que se dedicaban a los sistemas de información, ahora se pueden dedicar al cumplimiento del core del negocio, así como la apertura de una nueva opción para las entidades dedicadas a promover proyectos emprendedores al ofrecer la infraestructura cloud, como un valor agregado que descarga la responsabilidad de tener un área de sistemas para cada empresa al compartir estor recursos entre ellas. 


\section{Bibliografía}

1. + Publicidad \& marketing. (2010, 7 de abril). Cloud computing ya tiene casos de éxito en Colombia. En + publicidad \& marketing. [en línea] Disponible en: http:// maspublicidadymarketing.com/cloud-computing-yatiene-casos-de-exito-en-colombia/ (consulta: 4 de junio de 2010).

2. Allen, B. (2009, 24 de febrero). The goverment's effective migration to a cloud computing enviroment. Obtenido de Buzz Allen Hamilton: http://www.boozallen.com/ media/file/government-migration-to-cloud-computing. pdf

3. Amazon. (sf). Case Studies. En Amazon web services (en línea] Disponible en: http://aws.amazon.com/solutions/ case-studies/ (consulta: 27 de mayo de 2010).

4. Barboza Flores, S. (2008). Incubación de empresas de base tecnológica, la experiencia de Costa Rica. 2009. Costa Rica: Instituto Tecnológico de Costa Rica (TEC).

5. Beooklin, J. (2008, 2 de julio). Gartner: Seven cloudcomputing security risks. En Networkworld [en línea:] Disponible en: http://www.networkworld.com/ news/2008/070208-cloud.html (consulta: 13 de junio de 2009).

6. Camacho Pico, J. (1988). Incubadoras o viveros de empresas de base tecnologica: la reciente experiencia europea como referencia para las actuales y futuras iniciativas latinoamericanas. XII Congreso Latinoamericano sobre Espíritu Empresarial. Costa Rica: Instituto Tecnológico de Costa Rica (TEC).

7. Carr, N. (2008, 5 de noviembre). New York Times AWS Cloud Computing Mistake Cost \$240. En Green (low carbon) Data Center Blog. [en línea] Disponible en: http://www.greenm3.com/2008/11/nytimes-cloud-c. html (consulta: 3 de junio de 2010).

8. Clarck, T. (2010). Quantifying the Benefits of the RightScale Cloud Management Platform. En Rightscale [en línea] Diosponible en: http://www.rightscale.com/ info_center/white-papers.php (consulta: 8 de junio de 2010).

9. Cloud TP. (sf). Cloud Computing Success Stories. En cloud TP [en línea] Disponible en: http://cloudtp.com/cloudcomputing/cloud-computing-success-stories (consulta: 23 de mayo de 2010).

10. Consejo Nacional de Política Económica y Social (Conpes). (2005). Consolidación del sistema nacional de innovación, desarrollo tecnológico y emprendimiento en Colombia. Bogotá: DNP.

11. Consejo Nacional de Política Económica y Social (Conpes). (2009). Política nacional de ciencia, tecnología e innovación. Bogotá: DNP.

12. Dignan, L. (2008, 7 de abril). Cloud computing: A look at the myths. En Zdnet.com. [en línea] Disponible en: http://blogs.zdnet.com/BTL/?p=8409 (consulta: 25 de febrero de 2009).

13. Fontecilla, R. (2009, 13 de abril). Cloud Computing: $A$ Transition Methodology. Obtenido de Virtualization journal: http://virtualization.sys-con.com/node/886060

14. Gaw, P. (2008, 25 de julio). What's the Difference Between Cloud Computing and SaaS? [en línea] Disponible en: http://web2.sys-con.com: http://web2.sys-con.com (consulta: 13 de febrero de 2009).

15. Geelan, J. (2009, 21 de julio de 2009). The Top 150 Players in Cloud Computing. Obtenido de Virtualization Journal: http://virtualization.sys-con.com/node/770174

16. Golden, B. (2009, 18 de enero de 2009). The case against cloud computing Obtenido de Computerworld: http://www.computerworld.com/s/article/9126620/ The_case_against_cloud_computing_part_ one?intsrc=news_ts_head

17. Intel Corp. (sf). Moore's Law. [en línea] Disponible en: Intel http://www.intel.com/technology/mooreslaw/ (consulta: 12 de abril de 2009).

18. J, K. (2009). Understand Cloud Computing. Obtenido el 9 de febrero de 2009 de Web Hosting Blog, de http://blog. micfo.com/web-hosting-articles/understand-cloudcomputing

19. Johnson, B. (2008, 29 de septiembre). Cloud computing is a trap, warns GNU founder Richard Stallman. [en línea] Disponible en: guardian.co.uk: http://www.guardian. co.uk/technology/2008/sep/29/cloud.computing. richard.stallman (consulta: 13 de junio de 2009).

20. Knorr, E.; \& Gruman, G. (2008, 7 de abril). What Cloud Computing Really Means. En The New York Times.

21. Knorr, E.; \& Gruman, G. (2008). What Cloud Computing Really Means. En Inforworld [en línea] Disponible en: http://www.infoworld.com/article/08/04/07/15FEcloud-computing-reality_1.html (consulta: 9 de noviembre de 2008). 
22. Linthicum, D. (2010, 19 de marzo). The cloud's three key issues come into focus. En Infoworld. [en línea] Disponible en: http://www.infoworld.com/d/cloudcomputing/clouds-three-key-issues-come-focus-164 (consulta: 22 de marzo de 2010).

23. Lohr, S. (2001, 16 de abril). When Cloud Computing Doesn't Make Sense. En The New York Times [en línea] Disponible en: http://bits.blogs.nytimes. com/2009/04/15/when-cloud-computing-doesntmake-sense/ (consulta: 2 de junio de 2010).

24. Miller, M. (2009). Cloud computing. Web based applications that change the way you work and collaborate online. Indianapolis Indiana: QUE.

25. Narasimhan, B. (2009, 16 de abril). Cloud Computing Savings - Real or Imaginary? En CIO Blog [en línea] Disponible en: http://blog.appirio.com/2009/04/cloudcomputing-savings-real-or.html (consulta: 5 de junio de 2010).

26. Nauses, L. (2009). Practical Strategies for Moving "real organizatinos" towars Clous/Saas solutions. Cloud Computing journal.

27. Network World. (2008, 6 de enero). Cloud computing: Las TI como servicio. Network World. [en línea] Disponible en: $\quad$ http://www.idg.es/Comunicaciones/articulo. asp?id=191003 (consulta: 10 de octubre de 2008).

28. Perry, G. (2008, 28 de febrero). How Cloud \& Utility Computing Are Different. En gigaom.com [en línea] Disponible en: http://gigaom.com/2008/02/28/howcloud-utility-computing-are-different/ (consulta: 2 de febrero de 2009).

29. Perry, R.; Hatcher, E.; \& et al. (2009, 1 de septiembre). Force.com Cloud Platform Drives Huge Time to Market and Cost Savings. En TechRepublic.com/White Papers. [en línea] Disponible en: http://whitepapers. techrepublic.com.com/abstract.aspx?kw=idc+force. com\&docid=1188547\&tag=tr-left (consulta: 1 de junio de 2010).

30. Rebés, JM. (2008, 6 de enero). Network World. [en línea] Disponible en: http://www.idg.es/Comunicaciones/ articulo.asp?id=191003 (consulta: 10 de octubre de 2008).

31. Schadler, T. (2008, 29 de octubre). Talking to your CFO About Cloud Computing. En highlandmsp [en línea] Disponible en: www.highlandmsp.com/pdfs/CFO_ Cloud.pdf (consulta: 7 de junio de 2010). 
Anexo 1. Comparación del ROI utilizando Windows Azure

\section{Comparison of Windows Azure Platform vs. On-Premises for cesa}

In this section of the analysis, the Windows Azure Platform costs are quantified versus a comparable On-Premises solution (see below). Review and edit the default assumptions for On-Premises by clicking on this link:

Detailed Assumptions for On-Premises

Using the Windows Azure Platform Costs and Detailed Assumptions (above) for On-Premises solutions, comparing these two application delivery options results in the following advantages for the Windows Azure Platform:

\begin{tabular}{l|r|}
\hline $\begin{array}{l}\text { Windows Azure Platform Advantage - Three } \\
\text { Year Analysis Period }\end{array}$ & $\begin{array}{r}\text { Windows Azure } \\
\text { Platform vs. 0n- } \\
\text { Premises }\end{array}$ \\
\hline Net Savings with Windows Azure Platform & $\$ 178,311$ \\
\hline Total Windows Azure Platform Costs & $\$ 79,627$ \\
\hline Return on Investment (ROI) & $220.0 \%$ \\
\hline $\begin{array}{l}\text { Net Present Value (NPV) Savings (discount rate } \\
11.0 \% \text { ) }\end{array}$ & $\$ 160,076$ \\
\hline $\begin{array}{l}\text { Time it takes for the Windows Azure Platform (in } \\
\text { months) to recoup investment with savings when } \\
\text { compared to alternatives }\end{array}$ \\
*On-premises is configured with virtualization for comparison. \\
* Purchase cost for initia/ on-premises infrastructure is included.
\end{tabular}

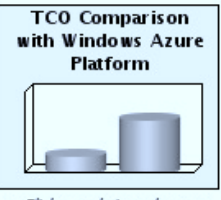

<Click graph to enlarge>

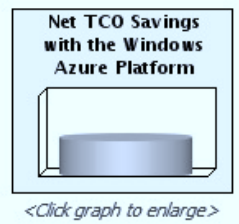

Windows Azure Platform vs. On-Premises (virtualized)

\begin{tabular}{|c|c|c|c|c|}
\hline \multicolumn{5}{|c|}{ Windows Azure Platform vs. On-Premises (virtualized) } \\
\hline $\begin{array}{c}\text { Windows Azure Platform Savings Over } \\
\text { Three Year Analysis Period }\end{array}$ & $\begin{array}{c}\text { Windows Azure } \\
\text { Platform }\end{array}$ & $\begin{array}{l}\text { On-Premises } \\
\text { (virtualized) }\end{array}$ & $\begin{array}{l}\text { Windows AzU } \\
\text { Savings vs. } 0\end{array}$ & \\
\hline Web / worker computing & $\$ 16,819$ & $\$ 54,486$ & $\$ 37,667$ & $69.1 \%$ \\
\hline Storage (non-relational) & $\$ 12$ & $\$ 12$ & $\$ 0$ & $0.0 \%$ \\
\hline Database computing, licensing and storage & $\$ 710$ & $\$ 23,469$ & $\$ 22,759$ & $97.0 \%$ \\
\hline Service Bus connections & $\$ 761$ & $\$ 0$ & $(\$ 761)$ & $0.0 \%$ \\
\hline Access Control transactions & $\$ 379$ & $\$ 0$ & $(\$ 379)$ & $0.0 \%$ \\
\hline Bandwidth & $\$ 56,412$ & $\$ 121,065$ & $\$ 64,653$ & $53.4 \%$ \\
\hline$\Pi$ administration and support & $\$ 4,448$ & $\$ 26,055$ & $\$ 21,607$ & $82.9 \%$ \\
\hline Facilities and overhead & $\$ 0$ & $\$ 25,935$ & $\$ 25,935$ & $100.0 \%$ \\
\hline Setup and delivery & $\$ 86$ & $\$ 6,916$ & $\$ 6,830$ & $98.8 \%$ \\
\hline Total Cost Over Three Year Analysis Period & $\$ 79,627$ & $\$ 257,938$ & $\$ 178,311$ & $69.1 \%$ \\
\hline Number of Instances / Physical Servers & 6 & 3 & & \\
\hline Average Total Cost per Month & $\$ 2,212$ & $\$ 7,165$ & $\$ 4,953$ & \\
\hline Net Present Costs (discount rate $=\mathbf{1 1 . 0} \%$ ) & $\$ 67,247$ & $\$ 227,324$ & $\$ 160,077$ & $70.4 \%$ \\
\hline
\end{tabular}

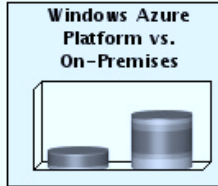

<Clok graph to enlarge>

Windows Azure

Platform

Advantages

On-Premises Details by Month

Sources of Default Metrics

On-Premises (virtualized) Details

The estimated costs of an equivalent On-Premises (virtualized) solution.

\begin{tabular}{|c|c|c|c|c|}
\hline $\begin{array}{c}\text { On-Premises Costs Over Three Year } \\
\text { Analysis Period }\end{array}$ & Year 1 & Year 2 & Year 3 & Total \\
\hline Web / worker computing & $\$ 41,478$ & $\$ 6,504$ & $\$ 6,504$ & $\$ 54,486$ \\
\hline Storage (non-relational) & $\$ 7$ & $\$ 2$ & $\$ 3$ & $\$ 12$ \\
\hline Database computing, licensing and storage & $\$ 13,301$ & $\$ 4,962$ & $\$ 5,206$ & $\$ 23,469$ \\
\hline Service Bus connections & $\$ 0$ & $\$ 0$ & $\$ 0$ & \$o \\
\hline Access Control transactions & $\$ 0$ & $\$ 0$ & $\$ 0$ & $\$ 0$ \\
\hline Bandwidth & $\$ 46,724$ & $\$ 36,341$ & $\$ 38,000$ & $\$ 121,065$ \\
\hline$\Pi$ administration and support & $\$ 8,550$ & $\$ 8,581$ & $\$ 8,924$ & $\$ 26,055$ \\
\hline Facilities and overhead & $\$ 8,644$ & $\$ 8,645$ & $\$ 8,646$ & $\mathbf{\$ 2 5 , 9 3 5}$ \\
\hline Setup and delivery & $\$ 6,916$ & $\$ 0$ & $\$ 0$ & $\$ 6,916$ \\
\hline Total Cost & $\$ 125,620$ & $\$ 65,035$ & $\$ 67,283$ & $\$ 257,938$ \\
\hline Average Number of Physical Servers & 3.0 & 3.1 & 3.1 & 3.1 \\
\hline Average Cost per Server Per Year & $\$ 41,873$ & $\$ 20,979$ & $\$ 21,704$ & $\$ 28,185$ \\
\hline Average Total Cost per Month & $\$ 10,468$ & $\$ 5,420$ & $\$ 5,607$ & $\$ 7,165$ \\
\hline Average Cost per Server Per Month & $\$ 3,489$ & $\$ 1,748$ & $\$ 1,809$ & $\$ 2,349$ \\
\hline
\end{tabular}

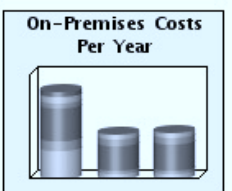

$\langle$ click graph to enlarge $>$

Next Steps

E-mail a report (registration required)

Learn more about the Windows Azure Platform

Click to view Windows Azure Platform Methodology document

Case Studies

Persistent Systems

Alinean 